\title{
Mid-latitude solar eclipses and their influence on ionospheric current systems
}

\author{
A. T. Tomás, H. Lühr, and M. Rother \\ Helmholtz-Zentrum Potsdam, Deutsches GeoForschungsZentrum (GFZ), Telegrafenberg, 14473 Potsdam, Germany
}

Received: 18 December 2008 - Revised: 7 September 2009 - Accepted: 29 September 2009 - Published: 8 December 2009

\begin{abstract}
Using CHAMP magnetic field data we study the behaviour of the geomagnetic field during two mid latitude eclipses on 21 June 2001 and 22 September 2006. The possible influence of the eclipses on different ionospheric current systems, as seen in the magnetic field measured by CHAMP, is discussed. It is expected that the blocking of solar radiation during an eclipse causes a reduction of the ionospheric conductivity and therefore has an effect on the different current systems. We address in particular the effects of the eclipses on the inter-hemispheric field-aligned currents and on the $\mathrm{S}_{q}$ current system. The two events studied occur under different seasonal conditions, e.g. June solstice and September equinox, therefore quite different aspects can be investigated. We find that the eclipses might affect the direction and intensity of the inter-hemispheric currents and possibly influence the direction of zonal winds, therefore changing the direction of the prevailing F-region dynamo currents. The eclipse in the Southern Hemisphere during September equinox caused inter-hemispheric currents similar to those observed in northern summer. Reverse inter-hemispheric currents were recorded after the end of the eclipse. A large variety of atypical currents was observed during the June event. Most of them might be related to a reversed F-region dynamo in the morning sector and an enhanced conductivity difference between the hemispheres. The eclipse in the south seems to enhance the June solstice conditions considerably.
\end{abstract}

Keywords. Ionosphere (Electric fields and currents; Midlatitude ionosphere)

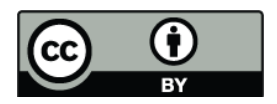

Correspondence to: A. T. Tomás (tomas@gfz-potsdam.de)

\section{Introduction}

Solar eclipses provide a unique opportunity to study the reaction of the ionosphere to the sudden blocking of solar radiation. In particular, the effects on the ionospheric current systems are still controversially discussed. In a previous work Tomás et al. (2008) investigated the effects of equatorial solar eclipses on the equatorial electrojet (EEJ) and found a local weakening of the EEJ which in most cases favoured the formation of a counter electrojet.

The work of Korte et al. (2001) showed that although the E-region electron density was markedly reduced during the 11 August 1999 eclipse, no significant change in $S_{q}$ current strength was detected. The authors suggest that the missing effect could be explained by considering a bypass of the current by field-aligned currents (FAC) to the Southern Hemisphere during the eclipse. The FAC system would then close in the E-layer through Pedersen currents. However, this effect was not confirmed using ground-base observations, and the suggestion was made that the closure of currents happened in the F-region, for which satellite observations are necessary.

Using CHAMP data, we present here a study of midlatitude eclipses and their effect on different ionospheric current systems.

One of the open questions concerns the effect of the blocking of solar radiation during the eclipse on the field-aligned current system. It is known that FACs are established when a change of local conditions cause an electrical potential difference between the hemispheres at the ionospheric footprints of a magnetic field line (VanSabben, 1966; Fukushima, 1991). When a magnetic flux tube has a higher electric potential on one end than the conjugate end, electric currents will flow along the field line to compensate this imbalance, which means that FACs will flow between the hemispheres. According to the authors, this electric potential difference reflects an asymmetry of the pattern and intensity of the $\mathrm{S}_{q}$

Published by Copernicus Publications on behalf of the European Geosciences Union. 

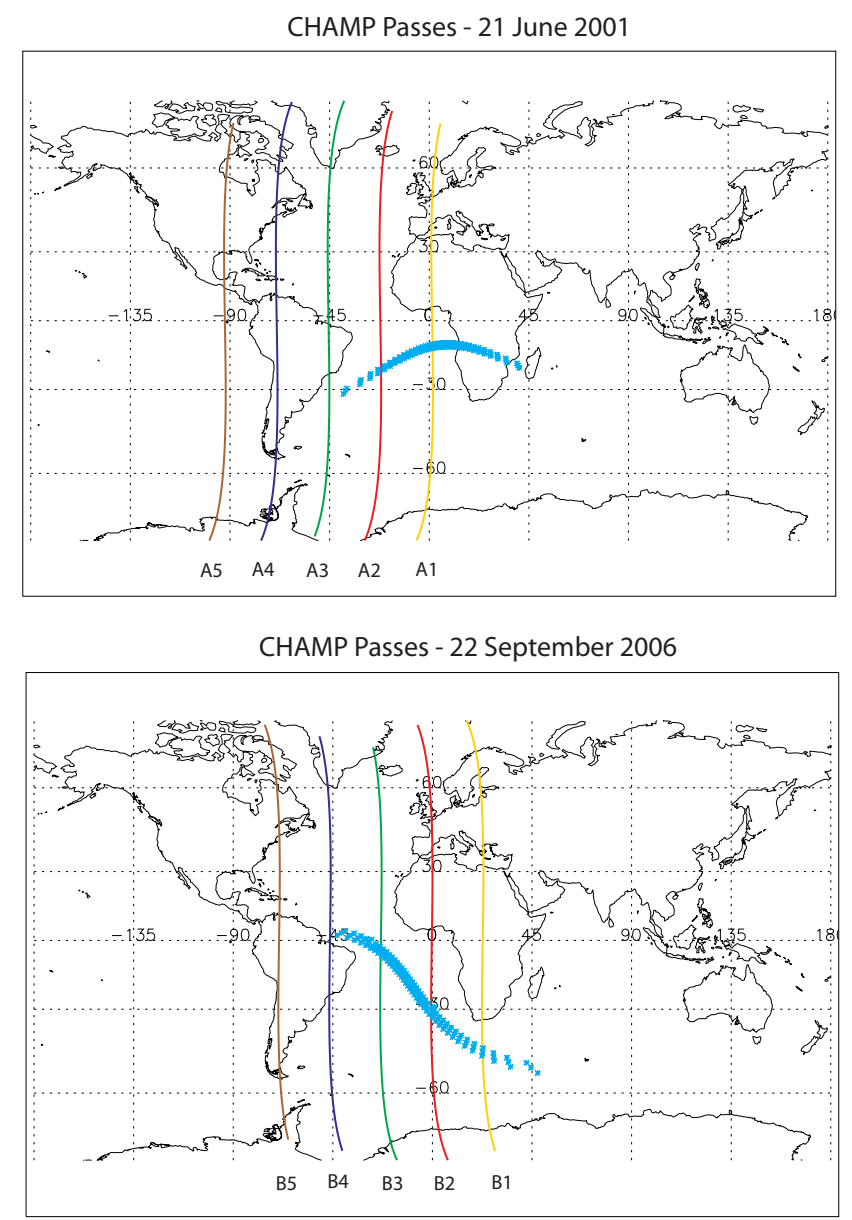

Fig. 1. World map projection for the 2 eclipses, showing the path of the umbral/antumbral shadow (cyan) and the CHAMP passes considered. The time for each pass (accordingly labelled) is indicated in Table 1.

current system. Fukushima (1991) suggests that the total amount of FAC in the ionosphere is greater during solstices than equinox since the potential differences between hemispheres should be greater at solstice.

Onwumechili (1997) mentions, among the possible causes for the electrical imbalance, a change in the distribution of the solar radiation or in the distribution of tidal forces and their associated winds. The FAC current density at ionospheric level, near the $S_{q}$ foci, is expected to be much less than $1 \mu \mathrm{A} / \mathrm{m}^{2}$ (Fukushima, 1991).

Recent studies of the inter-hemispheric field-aligned currents (IHFAC), based on Ørsted and ground-base data, give us some detailed information on the direction of the IHFAC in dependence of local time and season (Yamashita and Iyemori, 2002). The authors found that currents flow from the winter hemisphere to the summer hemisphere in the noon and dusk sector and in the opposite direction at dawn. The direction switches in April or May and in November, i.e. with a two months delay from the equinox and solstice phase. Studies of the seasonal variation of the diurnal and semidiurnal geomagnetic variations (Chulliat et al., 2005) has also found that the maximum of the $S_{q}$ current system does not occur during the June solstice.

Another interesting feature is the reaction of the F-region dynamo. The F-region dynamo drives a current system established due to the thermospheric zonal winds in the low latitude F-layer. The electric fields originating in the F-region are mapped to the E-layer along the field lines, as introduced by Risbeth (1971). The F-region dynamo currents have been observed by CHAMP, as variations in the $B_{y}$ component of the magnetic field (Lühr and Maus, 2006). The authors found that in the evening sector eastward winds cause vertical upward currents, which will then flow along field lines into both hemispheres, and close as equatorward Pedersen currents, while in the morning sector westward winds will cause currents in the opposite direction.

Our aim here is to address some of the open issues by making use for the first time of satellite observations to study the 3-D characteristics of eclipse-induced currents. The two considered events are regarded as very suitable since they occurred under quite different conditions concerning local time and season.

In the section to follow we first introduce the CHAMP data and the processing approach. We then present the observations for both eclipses and discuss the current features.

\section{Data and processing approach}

In this work we have focused on two mid-latitude eclipses on 21 June 2001 and 22 September 2006. Information concerning the solar eclipse characteristics was obtained from the NASA Solar Eclipse web page (http://sunearth.gsfc.nasa. gov/eclipse/eclipse.html). The ground tracks of the eclipses and the analysed CHAMP passes are shown in Fig. 1. For convenience of the reader each satellite pass is labelled, and the corresponding universal time (UT), as well as longitude (in degrees) and local time (LT) of the equator crossings are listed in Table 1.

For both eclipses we present the magnetic field components as measured by CHAMP. The data have been transformed into a Mean-Field-Aligned (MFA) coordinate system. As a reference for the orientation of the mean field we use the Potsdam Magnetic Model (POMME 3). Details of this model can be found in Maus et al. (2006). The rotation angles from the North-East-Center (NEC) frame to the MFA frame are calculated for every CHAMP position from POMME-3 and then applied to the actual measurements. The MFA system represents a local Cartesian frame in which the $\mathrm{z}$-axis is aligned with the average field direction, the $\mathrm{y}$-axis points in the east direction and is perpendicular to the magnetic meridian and the $\mathrm{x}$-axis completes the system. After having obtained the magnetic measurements in the MFA 
Table 1. Eclipse initial and final times. Location of the equator crossings of the CHAMP passes. Indicated are: Universal time (UT), longitude (in degrees) and Local Time (LT).

\begin{tabular}{|c|c|c|c|c|c|c|c|c|c|c|c|c|c|}
\hline \multirow[t]{2}{*}{$\mathrm{Ecl}$} & \multirow{2}{*}{ Date } & \multirow[t]{2}{*}{ Time } & \multicolumn{2}{|c|}{ Pass 1} & \multicolumn{2}{|c|}{ Pass 2} & \multicolumn{2}{|c|}{ Pass 3} & \multicolumn{2}{|c|}{ Pass 4} & \multicolumn{2}{|c|}{ Pass 5} & \multirow[b]{2}{*}{ LT } \\
\hline & & & UT & Geo. Long. & UT & Geo. Long. & UT & Geo. Long. & UT & Geo. Long. & UT & Geo. Long. & \\
\hline A & 21 Jun 01 & $10: 40-13: 25$ & $07: 26$ & $1.28 \mathrm{E}$ & $08: 59$ & $22.12 \mathrm{~W}$ & $10: 33$ & $45.52 \mathrm{~W}$ & $12: 07$ & $68.92 \mathrm{~W}$ & $13: 39$ & $92.31 \mathrm{~W}$ & $07: 25$ \\
\hline B & 22 Sep 06 & $10: 00-13: 25$ & 09:04 & $22.75 \mathrm{E}$ & $11: 36$ & $0.26 \mathrm{~W}$ & $13: 07$ & $23.27 \mathrm{~W}$ & $14: 39$ & $46.26 \mathrm{~W}$ & $16: 11$ & $69.29 \mathrm{~W}$ & $11: 37$ \\
\hline
\end{tabular}

system we first subtracted the field magnitude derived from POMME 3 from the $B_{z}$ component and then converted the three components into the $H, D, Z$ system. For the transformation we use:

$$
\begin{aligned}
H & =B_{x} \cos I+B_{z} \sin I \\
D & =B_{y} \\
Z & =B_{z} \cos I-B_{x} \sin I
\end{aligned}
$$

Where $B_{x}, B_{y}, B_{z}$ are the components in the MFA frame and $I$ is the inclination angle at CHAMP position. In observatory convention $D$ refers to the declination angle. Here it is the field component perpendicular to the magnetic meridian, as commonly used in ionospheric-magnetospheric studies. The $H D Z$ coordinate system facilitates best the comparison with ground-base data.

The magnetic field component interpreted for the estimation of the field-aligned currents is the $D$ component. To reduce the amount of small-scale structure in the estimated currents the component is smoothed by a pass filter with a 20 s cutoff period, which corresponds to a scale-length of $\sim 150 \mathrm{~km}$. The field-aligned-current density, $j$, is calculated based on Ampère's law:

$j=\frac{1}{\mu_{0}}\left(\frac{\partial B_{y}}{\partial x}-\frac{\partial B_{x}}{\partial y}\right)$

Since we consider only variations in the $B_{y}(D)$ component, and as we are interested in calculating the FAC density in the E-layer (at $110 \mathrm{~km}$ ), i.e. at the ionospheric footprint of the field lines, the previous equation becomes:

$j=\frac{1}{\mu_{0}} \frac{\Delta D}{\left(\beta_{1}-\beta_{2}\right) 111 \times 10^{3} \sin I}$

where $\mu_{0}$ is the vacuum permeability, $I$ is the inclination at the E-layer and $\beta_{1,2}$ are the Apex latitudes at $110 \mathrm{~km}$ altitude of consecutive measurement points. The Apex coordinate system maps the measurement position along the field lines down to the E-layer and is commonly used in ionospheric studies involving the calculation of field-aligned currents. For a detailed description of these coordinates see Richmond (1995).

\section{The eclipse events}

\subsection{The 21 June 2001 eclipse}

The total solar eclipse of 21 June 2001 was visible in a narrow corridor in the Southern Hemisphere. It started at 10:40 UT (at $30.63^{\circ} \mathrm{S}$ latitude and $37.93^{\circ} \mathrm{W}$ longitude) and ended at $13: 25 \mathrm{UT}\left(19.93^{\circ} \mathrm{S}\right.$ latitude and $40.07^{\circ} \mathrm{E}$ longitude). The solar flux index (F10.7) indicated high solar activity on this day with a value of 200.3. The geomagnetic activity index $\left(K_{p}\right)$ was 2o from 09:00 UT to 12:00 UT and $2+$ from 12:00 UT to 15:00 UT, indicating low geomagnetic activity.

The CHAMP magnetic field measurements are plotted in Fig. 2. The different passes are identified by the same colour scheme and letter code as in Fig. 1. As noted in Table 1 the samples are taken in the morning sector, all close to 07:30 local time.

The top panel contains the $H$ component. The first two passes (A1, yellow and A2, red) show counter-electrojet (CEJ) profiles, with a positive peak around the equator, while the third pass (A3, green) shows a perturbed profile with no clear CEJ or EEJ feature. The last two passes (blue and brown) show regular EEJ profiles although with small peakto-peak variations. This type of EEJ variation is consistent with predictions of the climatological models EEJM-1 concerning the longitudinal dependence of the EEJ diurnal variation for the different seasons (Lühr et al., 2008). The authors found that around the June solstice, between $80^{\circ} \mathrm{W}$ and $90^{\circ} \mathrm{W}$ longitude, the EEJ changes from a CEJ, i.e. westward current, to normal EEJ at this local time. The analysed passes cover the same longitudinal and local time range. Therefore, we conclude that the observed switch in current direction is a typical EEJ variation and is not associated with the eclipse.

The middle panel shows the variation of the $D$ component. The first two passes show small variations around the equator and show no indications of FAC. The following pass (A3, green) shows a decrease around the equator, followed by a profile with small variations in the Northern Hemisphere, which seems to be related to the variations in the $H$ component. The subsequent pass (A4, blue) shows a strong positive deflection around the equator, indicating an inter-hemispheric FAC flowing upward in the northern and downward in the Southern Hemisphere. In the last pass (A5, brown) a return to the initial situation is seen. 

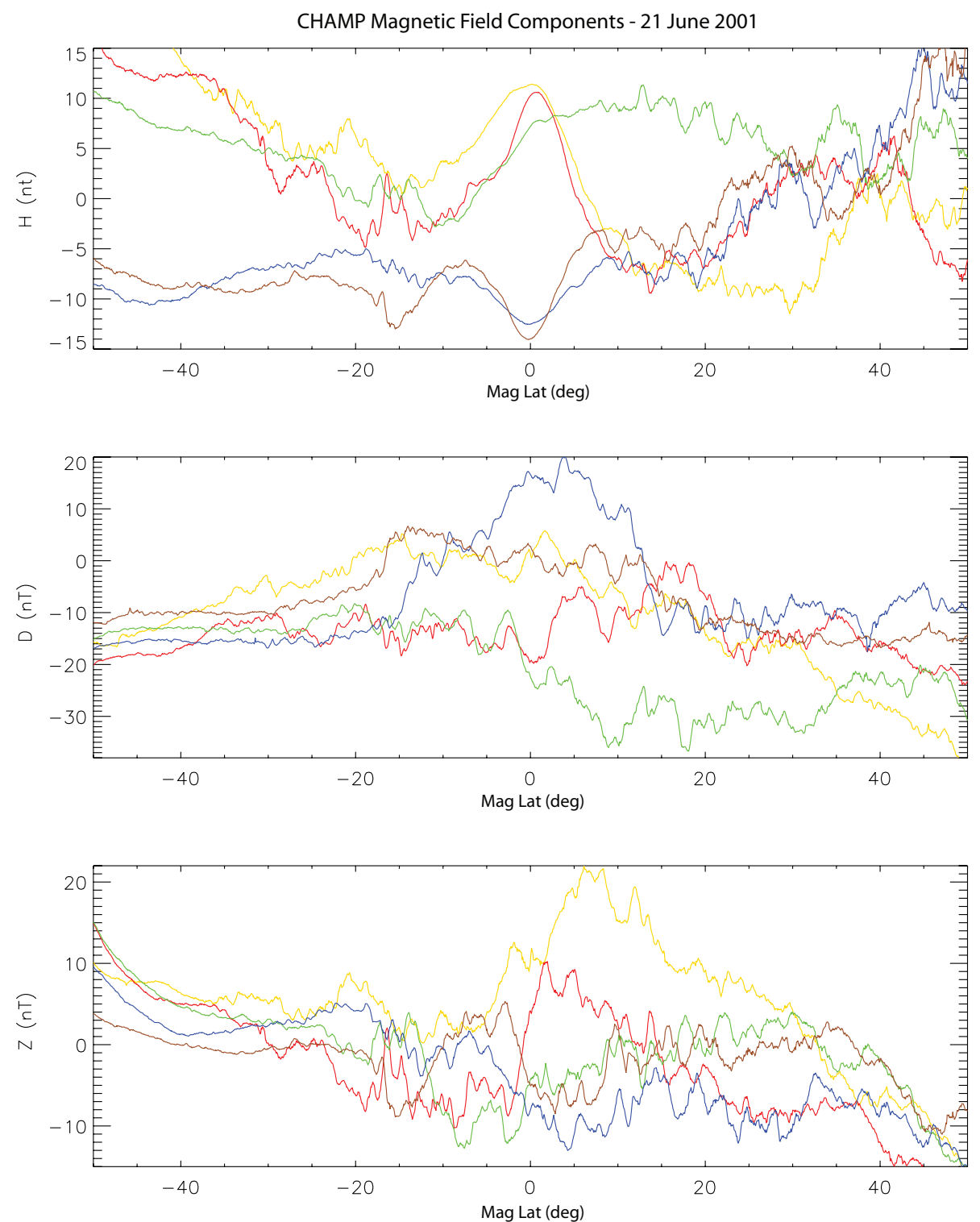

Fig. 2. Variation of the $H, D$, and $Z$ magnetic field components versus corrected magnetic latitude. The same colour code as in Fig. 1 is used.

The bottom panel shows the $Z$-component, with the two first passes exhibiting reversed profiles, which are associated with the CEJ seen in the $H$-component. The last passes show the normal positive/negative variation at the equator. Here again the green curve is abnormal.

\subsection{The 22 September 2006 eclipse}

The solar eclipse of 22 September 2006 was an annular solar eclipse. It started near the dip equator at 10:00 UT, at the Brazilian coast and proceeded in the south-east direction, through the South Atlantic, ending at 13:25 UT. Its path covered a region in latitude from $3.35^{\circ} \mathrm{N}$ to $51.93^{\circ} \mathrm{S}$ and in lon- gitude from $41.07^{\circ} \mathrm{W}$ to $44.53^{\circ} \mathrm{E}$. During this day the F10.7 index was 72.3 , indicating low solar activity and the $K_{p}$ index was $0+$ during the eclipse, indicating very calm geomagnetic conditions.

In Fig. 3 we can see the variation of the magnetic field components, $H, D$ and $Z$ (as explained in Sect. 2) versus the magnetic latitude. The different passes are identified using the same colour and letter scheme as in Fig. 1. In this case CHAMP scanned the 11.5 LT sector.

The $H$ component profiles (top panel) show the typical magnetic field signature of the equatorial electrojet (EEJ) with a negative deflection around the equator. Less typical is the first pass (B1, yellow, before eclipse), where the EEJ 

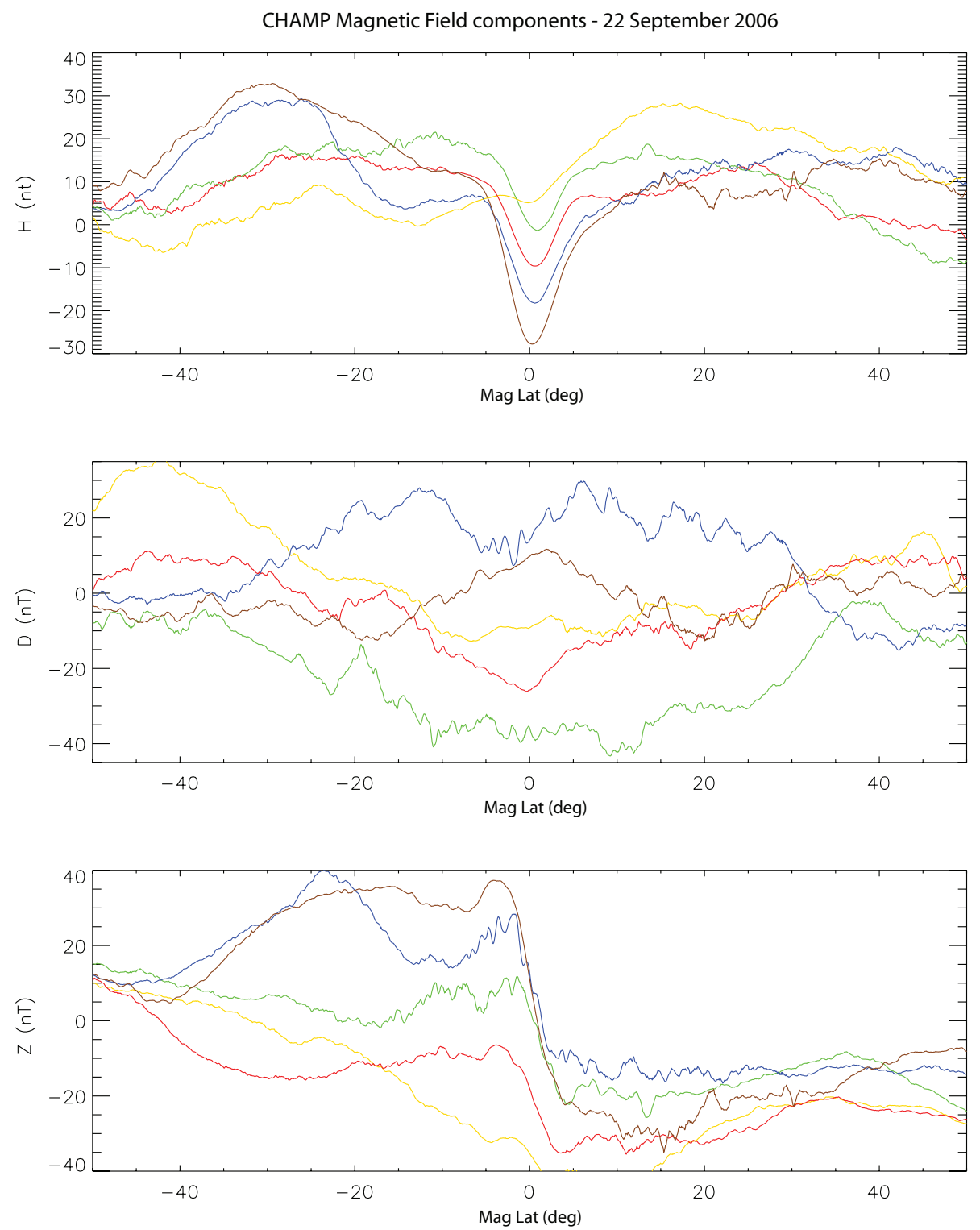

Fig. 3. The same as Fig. 2, but for the 22 September 2006 event.

profile is not so clear. Since these measurements were taken before the eclipse we do not further consider this pass.

In the $D$ component (middle panel) one can see small variations in the second (red) and the last (brown) passes, showing no indications of prominent field-aligned currents. On the contrary, the two intermediate passes (green and blue) show the typical signature of the IHFACs (unipolar deflection of $D$, symmetrical about the magnetic equator). On the B3 pass (green) the decrease in the Southern Hemisphere followed by an increase in the Northern Hemisphere reveals a FAC from the southern to the Northern Hemisphere. On the consecutive pass (B4) the reverse situation is observed, with the FAC going from north to south.
The $Z$ component (last panel) shows the normal step-like variation associated with the EEJ on all passes.

Opposed to the Northern Hemisphere the various passes exhibit quite different deflections in $\mathrm{Z}$ in the Southern Hemisphere.

As an aid for the proper interpretation of the eclipse related effects we present in Fig. 4 the annual averages of the $\mathrm{H}$ and $\mathrm{Z}$ latitude profiles as observed by CHAMP in the local time sectors $7.5 \mathrm{LT}$ and $11.5 \mathrm{LT}$. The average $D$ curves are practically flat. The profiles in the morning sector (top panel) show only small variations over latitude. In particular the EEJ causes a deflection of only a few nano Tesla. This indicates that the regular and counter-electrojet almost balance 

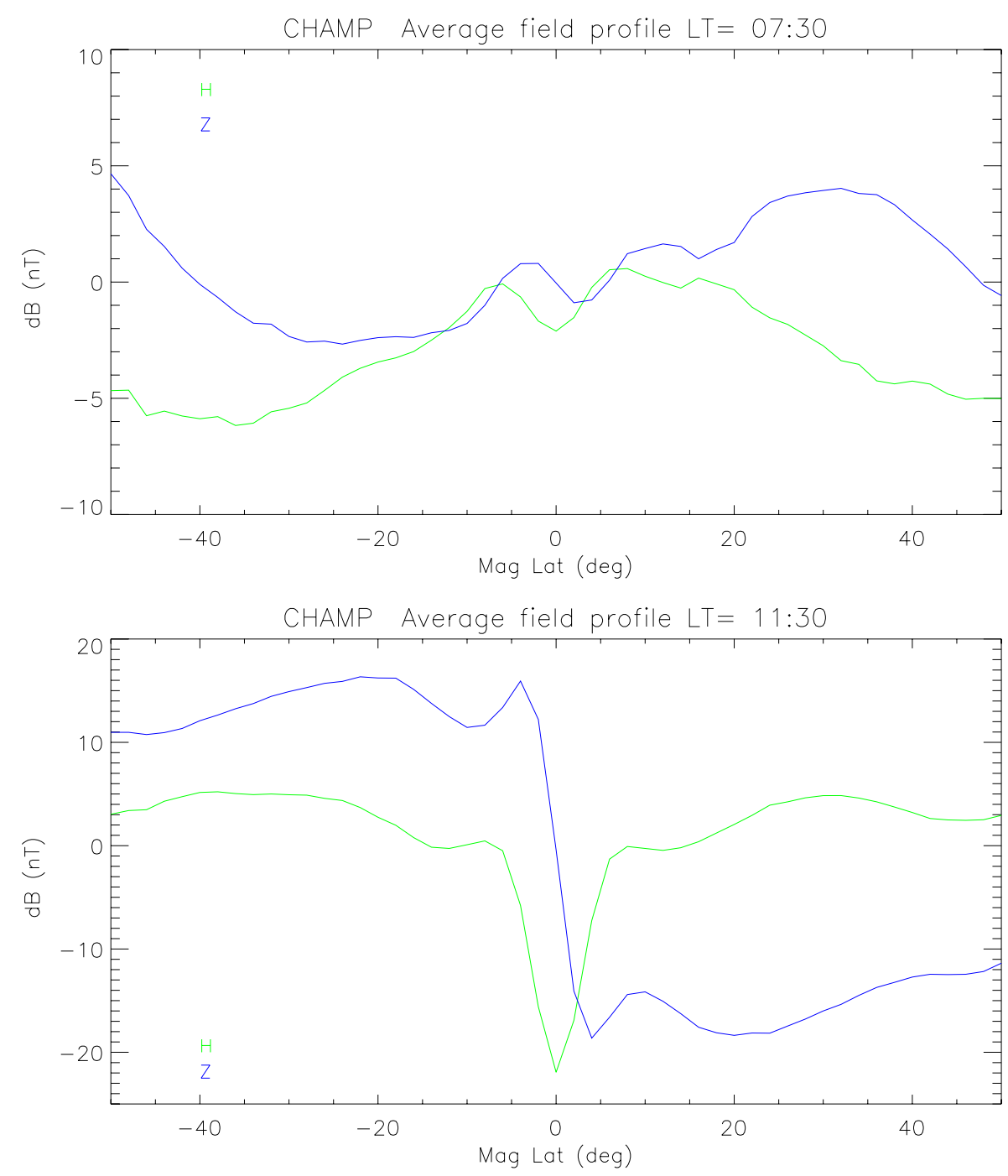

Fig. 4. Annual averages of the $H$ and $Z$ latitude profiles, as observed by CHAMP in the local time sectors = 07:30 LT (top) and 11:30 LT (bottom).

each other in the considered LT sector, when averaged for all longitudes, as was done for Fig. 4.

Distinct latitude profiles are obtained by CHAMP for the 11.5 LT sector. The $H$ component exhibits a symmetrical curve about the magnetic equator with a prominent negative deflection caused by the EEJ. Opposed to that $Z$ varies antisymmetrically with respect to the dip equator. The curves of both components reflect primarily the magnetic effects of the EEJ and $S_{q}$ current systems. For deriving the annually averaged curves we also represented the main field by the POMME 3 model. For that reason, the curves shown here can be used as reference for the individual observations.

\section{$4 \quad$ Interpretation of current features}

During both eclipse events we observe some magnetic field deflections on passes close to the shadow period which are quite different from the typical latitudinal variations as shown in Fig. 4. Here we interpret them in terms of currents and see how they are related to the shadow zone.

For the event of 22 September 2006 we observe in the $D$ component on pass B3 (green) a significant negative deflection at low latitudes and one orbit later (B4, blue) an antisymmetric positive disturbance (see Fig. 5). These east/west field excursions can be interpreted as field-aligned currents flowing from one hemisphere to the other, as mentioned before. For the evaluation of the FAC intensity we used Eq. (3). Since the FAC density depends on altitude, we decided to determine the current values at the ionospheric footprints 


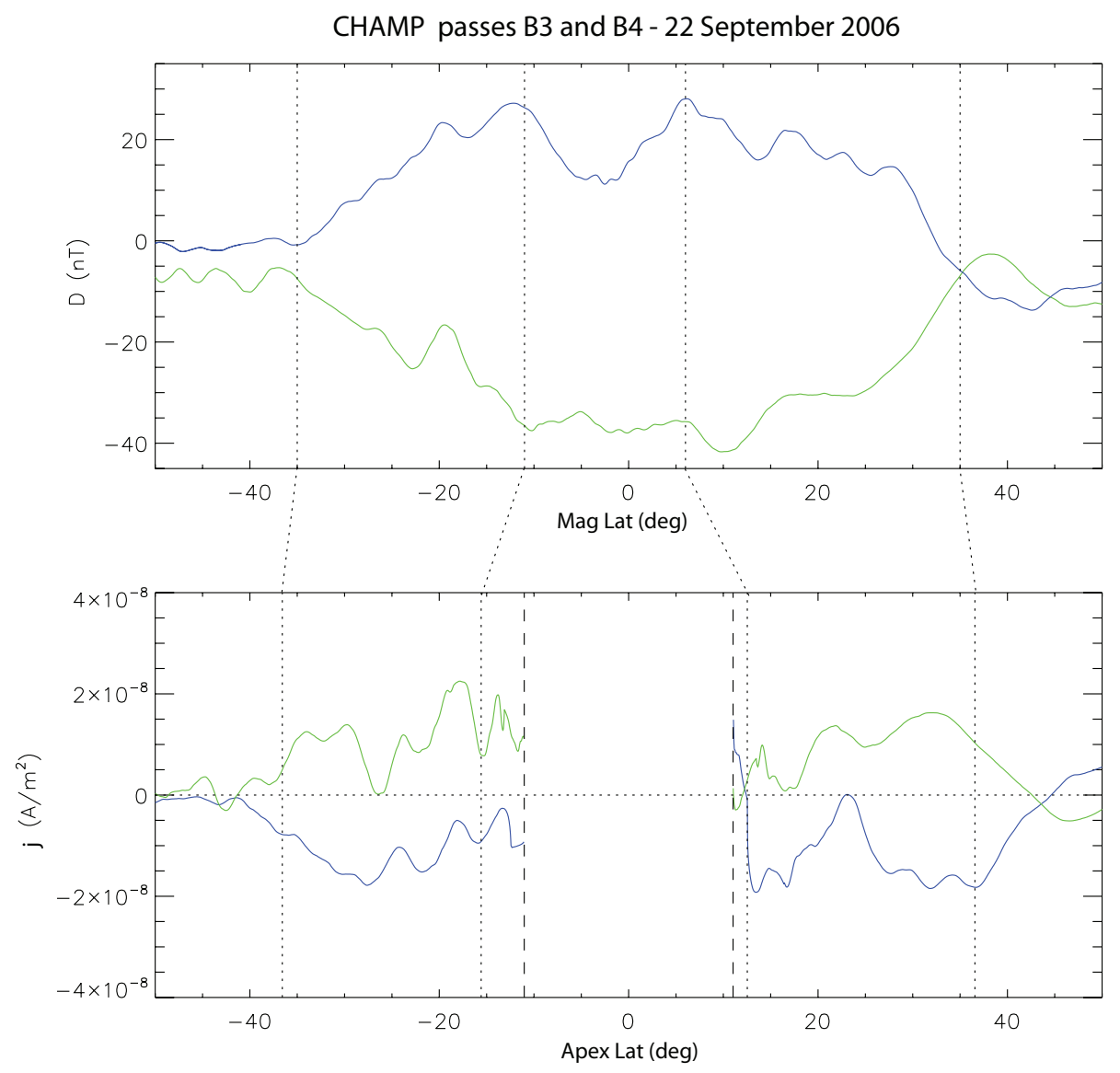

Fig. 5. Variation of the $D$ component (top) and corresponding field-aligned current density at E-layer altitude (bottom) for the latitude range of interest (dotted lines), for the 22 September 2006 solar eclipse. The unsampled E-region latitude gap is indicated by the dashed lines.

$(110 \mathrm{~km})$. The lower panel of Fig. 5 shows the derived current densities at this level. We obtain currents in the direction of the field, i.e. from Southern to Northern Hemisphere, of order $0.01 \mu \mathrm{A} / \mathrm{m}^{2}$ on pass B3 and an opposite current of comparable strength on the subsequent pass, B4.

In order to bring the observed currents into the context of the eclipse, Fig. 6 illustrates the footprints of the FACs together with the shadow contours during pass 3 . As can be seen, the upward directed FAC in the Southern Hemisphere originates from the trailing region behind the shadow zone. The opposite FAC observed on pass B4 occurs more than one hour after the end of the eclipse and far from the shadow. It may be interpreted as a reconfiguration effect of the thermospheric winds, driving the $\mathrm{S}_{q}$ system, after the event. The latitude range over which the FACs are detected ranges in both passes and hemispheres from about $15^{\circ}$ to $35^{\circ}$ magnetic latitude.

During the other event on 21 June 2001 we observe a larger variety of current features. When starting with the later pass, A4 (blue), we find a similar positive deflection in the $D$ component as in B4 (see Fig. 7). This is interpreted as an inter- hemispheric FAC from north to south. From the lower frame of Fig. 7 we can, however, read that the current density is much higher in this case and the footprint area is restricted to latitudes below $20^{\circ}$ magnetic latitude. The low latitude limit cannot be sensed by CHAMP due to its orbital altitude. Also these IHFACs are observed far behind the shadow zone (see Fig. 10).

On the previous passage, A3 (green), CHAMP passes right through the core shadow. Particular magnetic field deflections indicate the presence of several special ionospheric currents. The magnetic variations observed on this pass are repeated in Fig. 8. The $D$ component in the top panel shows a step-like change from zero in the Southern to negative in the Northern Hemisphere when crossing the magnetic equator. The observed negative slope can be interpreted as a passage through a vertical current sheet. From the change of the $D$ component between $-2^{\circ}$ and $8^{\circ}$ magnetic latitude we can deduce an upward directed sheet current density, $J_{v}=10 \mathrm{~mA} / \mathrm{m}$.

The vertical current at the equator has to close somewhere. We suggest that it is routed along field lines to the E-layer and closed there. The closure current should give in 


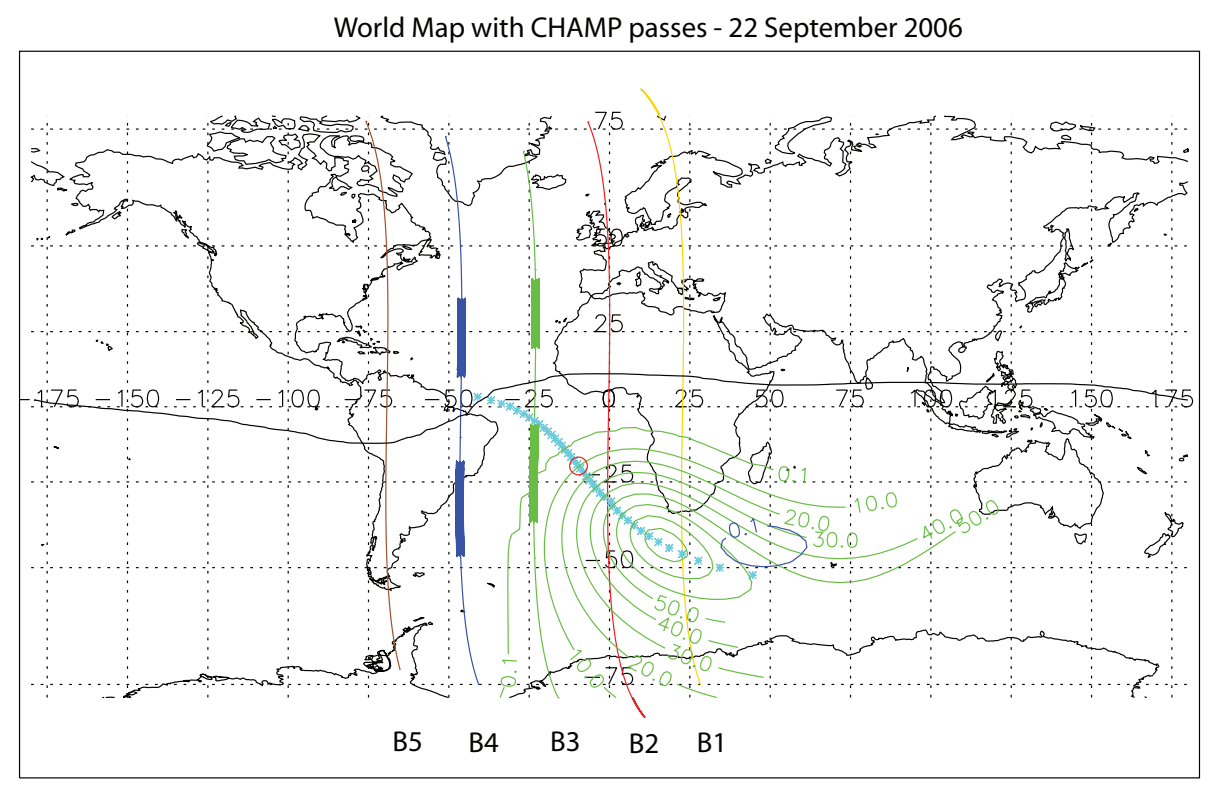

Fig. 6. World map projection showing the path of the eclipse (cyan), the CHAMP passes considered and the latitude of the magnetic equator (solid line). The bold parts of passes B3 and B4 indicate the location of the FAC regions. Contours indicate the expected shadow distribution at the time of the dip equator crossings of passes B3 and B4 (green and blue, respectively). The red circle indicates the closest approach for the $\mathrm{B} 2$ pass.

return a magnetic signature in the $D$ component detectable by CHAMP. An inspection of Fig. 8, top panel, reveals that there is no such signature in the Southern Hemisphere but a persistant negative deflection of about $15 \mathrm{nT}$ is observed between $10^{\circ}$ and $30^{\circ}$ magnetic latitude in the Northern Hemisphere. This is consistent with an equatorward Pedersen current in the E-layer that can constitute the current closure. The envisaged current configuration is depicted in Fig. 9. From the magnetic deflection we estimate a Pedersen current of at least $7 \mathrm{~mA} / \mathrm{m}$.

Also the $H$ and $Z$ components show particular deflections during the eclipse pass. We prefer to start the interpretation with the $Z$ component because it varies continuously through the current sheet and is thus less dependent on its height (see Fig. 8). Between $-13^{\circ}$ and $-8^{\circ}$ magnetic latitude there is a steep negative deflection. This is indicative of a passage over a narrow eastward current. Subsequently, we find a more gentle field increase, suggesting a wider westward current in the Northern Hemisphere. In order to validate our impressions on ionospheric currents we performed a few model calculations. A strip of the eastward current was assumed to flow in the southern hemispheric F-layer and a westward current in the northern hemispheric E-layer.

The calculated magnetic fields at CHAMP altitude are compared with observations. We varied the width and the position of the current sheets, as well as the current density, until we obtained a reasonable agreement with the observations. The modelled magnetic field deflections are over- plotted on Fig. 8. Dashed vertical lines mark the latitudinal boundaries of the modelled current sheets. The predicted signal fits both the $Z$ and $H$ observations reasonably well. To obtain that agreement we had to use for the narrow eastward current a density of $16 \mathrm{~mA} / \mathrm{m}$ within the magnetic latitude band $-13^{\circ}$ to $-8^{\circ}$ and for the wider westward current a density of $7.8 \mathrm{~mA} / \mathrm{m}$ distributed over the magnetic latitude $0^{\circ}$ to $30^{\circ}$.

\section{Discussion}

In this study we report about satellite observations made during total solar eclipses at mid latitudes. The two events are quite specific in that they occur under quite different conditions. This allows to address a range of different issues. The earlier event, 21 June 2001, takes place at solstice in the winter hemisphere under solar maximum conditions. CHAMP samples the effects occurring in the dawn sector (07:25 LT). Conversely, the later event, 22 September 2006, occurs at equinox during solar minimum, and CHAMP takes readings close to local noon (11:37 LT). When discussing the derived current systems we should have these differences in mind.

We will start with the event on 22 September 2006 because the effects can be explained in a straight forward manner. The prominent features observed are the inter-hemispheric field-aligned currents in the wake of the eclipse. On pass B3 CHAMP senses an IHFAC from south to north. Such a feature is typical at noon time for solstice conditions where 


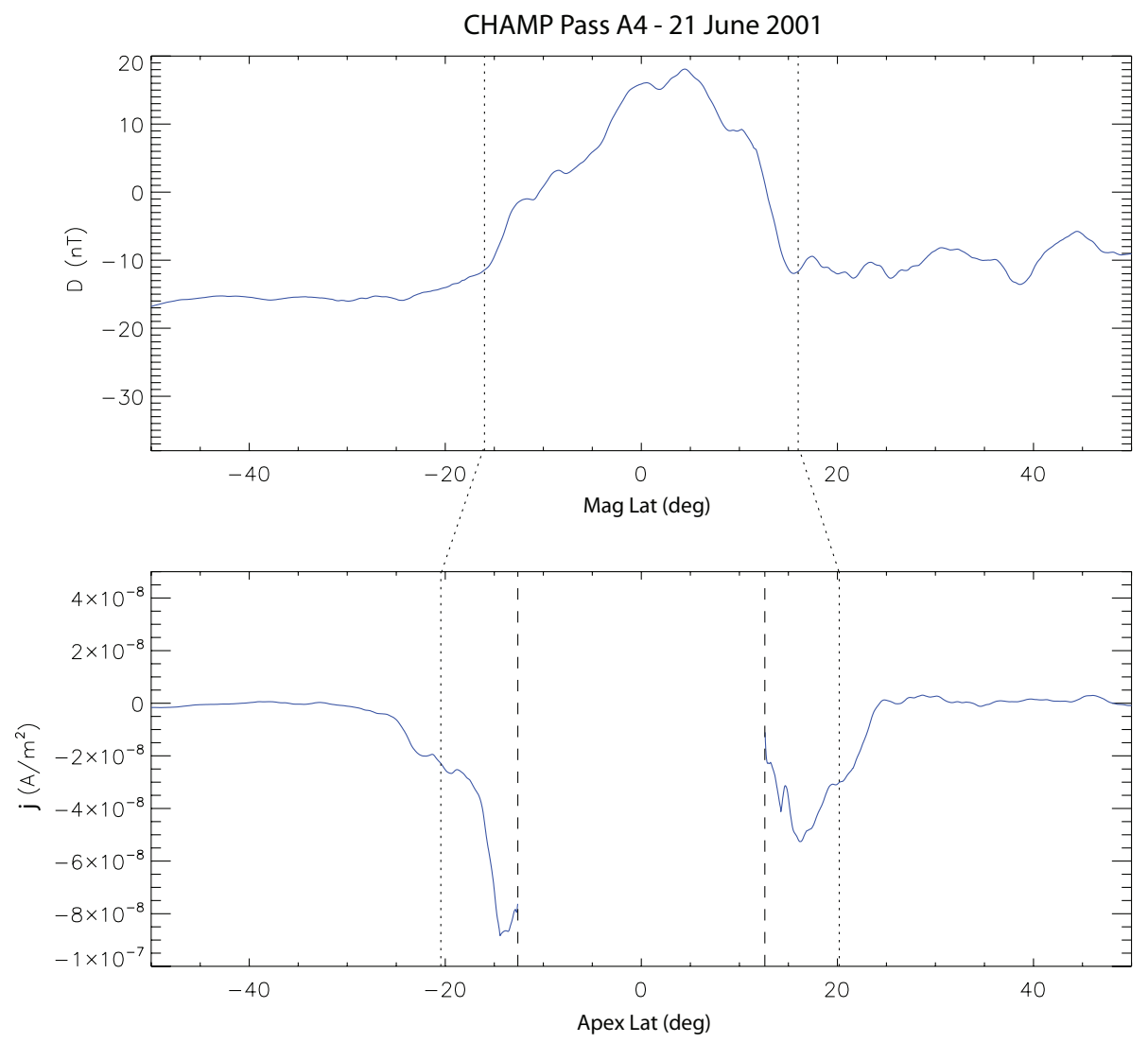

Fig. 7. The same as Fig. 5, but for the 21 June 2001 solar eclipse.

FACs flow out of the focus of the $\mathrm{S}_{q}$ system from the winter to the summer hemisphere (e.g. Yamashita and Iyemori, 2002). Although this event takes place at equinox the solar eclipse resembles southern winter conditions for a while. The height-integrated current density flowing between the ionosphere amounts to $20 \mathrm{~mA} / \mathrm{m}$. Yamashita and Iyemori (2002) obtain an average current density of $\sim 12 \mathrm{~mA} / \mathrm{m}$ for the summer months in 1999 (June to September), using the Ørsted data.

During the subsequent pass B4 (93 min later) a FAC in opposite direction but with similar strength is observed. This may reflect the return of the wind system to the equinox configuration.

The dominant mid-latitude current system at day time is the $\mathrm{S}_{q}$ system. It comprises ionospheric currents circulating around the focus at about $35^{\circ}$ magnetic latitude near noon. They flow counter-clockwise in the northern and clockwise in the Southern Hemisphere. Unperturbed $S_{q}$ currents manifest themselves in the CHAMP $Z$ component as more or less latitude independent positive deflections in southern and negative in the Northern Hemisphere (see Fig. 4). During the event on 22 September 2006 CHAMP passes the $\mathrm{S}_{q}$ system shortly before noon, therefore the $Z$ component observations are well suited to judge the strength of the current system. When going through the $\mathrm{Z}$ traces in chronological order we find the following deviations in the Southern Hemisphere (see Fig. 3). On B2 (red), the pass closest to the core shadow (see Fig. 6 red circle) there is a negative deflection centred at $-30^{\circ}$ magnetic latitude, which indicates a weakening of the $S_{q}$. The subsequent pass (green) reflects an almost normal curve with a slight depletion. A larger positive excursion of the blue curve (B4), centred at $-25^{\circ}$ magnetic latitude, signals a strongly enhanced $\mathrm{S}_{q}$ system with the focus displaced equatorward. Finally, during B5 the $\mathrm{S}_{q}$ is still strong. In the Northern Hemisphere the $Z$ component shows little structure. Thus the eclipse in the south has no significant influence on the $\mathrm{S}_{q}$ vortex structure in the north.

In Fig. 5 we have shown that the $D$ component deflections can be interpreted as inter-hemispheric currents. At the footprint of the downward current there is an accumulation of positive charges expected. In case of pass B4 IHFACs flow into the Southern Hemisphere. Such a pile-up of charge will setup a radial electric field which drives a clockwise Hall current vortex in the Southern Hemisphere thus strengthening the $S_{q}$ system. In Fig. 3 we actually find the peak in B4 $Z$ component at $-25^{\circ}$ magnetic latitude, which is close to the centre of the incoming FAC latitude at $-26^{\circ}$ (cf. Fig. 5). 

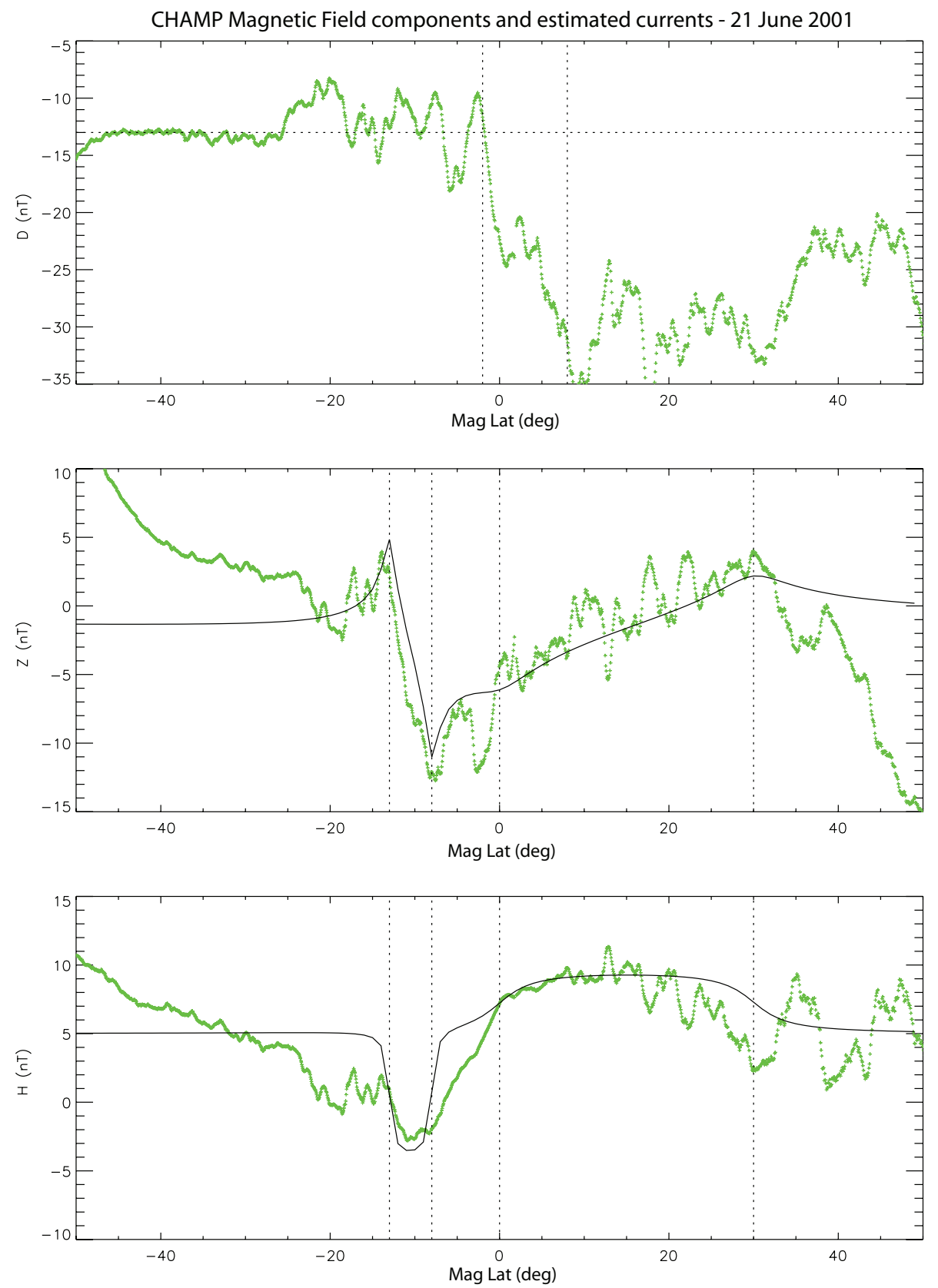

Fig. 8. Magnetic variations of the $D$ (upper panel), $Z$ (middle panel) and $H$ (lower panel) components as measured by CHAMP. The estimated profiles due to modelled currents in the indicated regions (dotted lines) are shown by the black lines.

When looking at the signal evolution in the $D$ component (see Fig. 3) it is obvious that the IHFAC from south to north (negative deflection) starts already during pass B2 (red) intensifies during B3 (green) then switches from north to south (blue pass) after which it slowly fades away (brown pass). This temporal evolution of FACs is in line with the modifications of the $\mathrm{S}_{q}$ system in the Southern Hemisphere while no local changes are recorded in the north. Obviously, the ionospheric conductivity in the north is high enough for preventing charge accumulations.
Another thing to note is the rather long time it takes for the $\mathrm{S}_{q}$ currents in the south to return to normal configuration. The IHFACs out of the eclipse region only start when the shadow is almost overhead and peak in the wake of it. The restoration of the hemispheric imbalance peaks several $(\sim 3)$ hours after the core shadow had passed the noon meridian, and even $5 \mathrm{~h}$ later and $70^{\circ}$ in longitude away this rebounce process is still going on. This implies that the thermospheric winds driving the $S_{q}$ current system have a large inertia. 
Now turning to the event on 21 June 2001, where CHAMP is sampling the morning sector (07:25 LT orbit). In this case the moon shadow is reducing further the solar insulation in the winter hemisphere, thus augmenting the seasonal conductivity difference. As illustrated in the previous chapter a variety of different currents are detected by CHAMP on its pass A3 which leads right through the core shadow (see Fig. 10). We regard the vertical upward current as deduced from the negative slope of the $D$ component in Fig. 7, as a key element for the interpretation of the whole current system. Such a current system is indicative of the action of the F-region dynamo (Risbeth, 1971; Lühr and Maus, 2006). Zonal winds at low latitudes cause a charge separation in vertical direction and set up a current system as illustrated in Fig. 9 (Heelis, 2004; Lühr and Maus, 2006). In our case we need an eastward wind to drive the observed upward current. This is somewhat unexpected since during the morning hours typically westward winds are prevailing in the thermosphere (Drob et al., 2008). The current driven by the F-region dynamo is routed along the field lines to the E-layer for closing as Pedersen current. The magnetic signature in the $D$ component (cf. Fig. 7) (persistent negative deviation) suggests a current closure exclusively in the sun-lit Northern Hemisphere and nothing in the winter and eclipse shaded hemisphere.

The E-region electric field caused by the equatorward flowing Pedersen current will set up a westward flowing Hall current. Such a current can be deduced from the variations in the $H$ and $Z$ components (cf. lower frames in Fig. 7). Our simple model calculation in Sect. 4 yielded for the westward current a sheet current density of $7.8 \mathrm{~mA} / \mathrm{m}$. In comparison to that a current density of at least $7 \mathrm{~mA} / \mathrm{m}$ was estimated for the Pedersen closure current. This match in strength supports our idea of Hall and Pedersen currents driven in the Northern Hemisphere by the F-region dynamo. It should be noted, however, that at this low latitude typically an eastward current is observed as part of the $\mathrm{S}_{q}$ system. This reverse current comes from the zonal wind at the magnetic equator in abnormal direction. As an explanation we may speculate that the switch from eastward to westward wind, occurring typically at 05:00 LT during June solstice, is delayed due to the solar eclipse in the morning sector by about three hours.

Not so easy to explain is the intense narrow eastward current at low southern latitudes $\left(\sim-10^{\circ}\right.$ magnetic latitude). It resembles in many respects the magnetic signature of the equatorial electrojet (cf. Fig. 7, bottom panel) but it is clearly displaced from the Cowling channel at the dip equator. A feature to note is that the peak-to-peak amplitudes of the deflections are clearly larger in the $Z$ component than in the $H$ component. This implies that CHAMP is passing through part of the current carrying volume only slightly above the current centre. The sheet current strength in a sheet can, for example, be estimated from the latitudinal gradient of the vertical component:

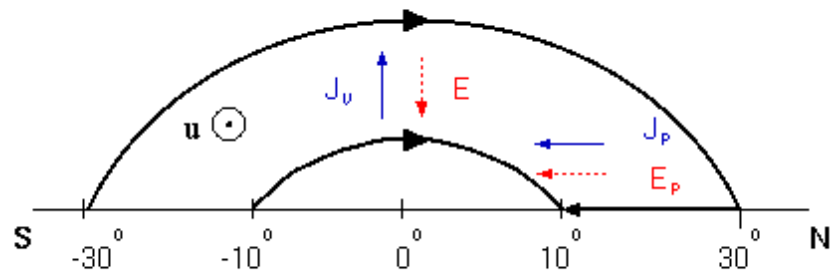

Fig. 9. Schematic representation of the vertical currents driven by the F-region dynamo and the Petersen closure currents in the Elayer.

$J=-\frac{4 \pi}{\mu_{0}} \frac{a^{2}+h^{2}}{4 a}\left(\frac{d Z}{d \theta}\right)_{\text {cur centre }}$

where $a$ is the latitudinal half-width of the current tube, $h$ the measurement height above the centre and $d \theta$ the latitudinal increment in meters.

When applying the current density derived from Eq. (4) to a current sheet in the E-layer we predict a too large deflection in the $H$ component. For reconciliating the $Z$ and $H$ variations we have to assume that CHAMP passed through the current ( $\sim 450 \mathrm{~km}$ orbit height). This has little effect on the $Z$ deflections but reduces the $H$ signature which is depending on the ratio of currents above and bellow CHAMP. For a current centre $\sim 50 \mathrm{~km}$ below CHAMP we obtain the best fit to the observed variation, as seen in Fig. 8.

How can we explain this confined channel carrying significant currents in the F-region? Our suggestion is that they are pressure gradient and gravity-driven currents (Lühr et al., 2003; Maus and Lühr, 2006). At the time of the peak deflection in H CHAMP passed a flux tube of high pressure. Electron temperature measurements (although not fully calibrated) attained a peak value well above $3000 \mathrm{~K}$. Also the ion temperature is expected to be enhanced here. This large temperature in the F-region is a typical signature known as the morning overshoot. The eastward current below CHAMP implies a steep plasma pressure (temperature) decrease towards lower altitudes. This can probably be explained by the eclipse effect.

About $10 \mathrm{~mA} / \mathrm{m}$ can be devoted to the pressure gradient currents. The rest we associate with gravity-driven currents. These currents are always directed eastward. Their strength depends primarily on the electron concentration:

$\mathbf{j}_{\mathbf{g}}=n_{e} m_{i} \mathbf{g} \times \mathbf{B} \frac{1}{B^{2}}$

When assuming oxygen ions for the mass, $m_{i}$, we need a total electron content, TEC $~ 50$ TECU, for explaining the remaining height-integrated current density of $6 \mathrm{~mA} / \mathrm{m}$. This is a reasonable value for the ionisation anomaly region. This eastward F-region current may also help to close the $\mathrm{S}_{q}$ current vortex in the Southern Hemisphere during the midlatitude eclipse. 


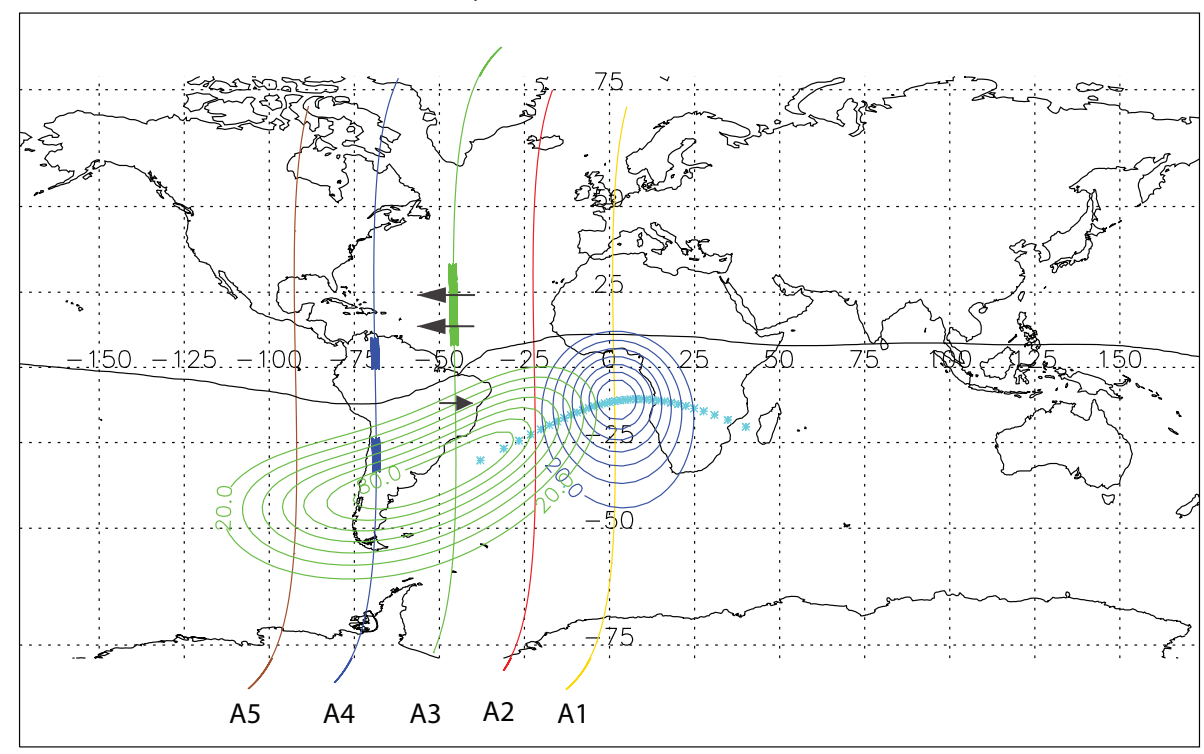

Fig. 10. World map projection for the 21 June 2001 eclipse, showing the path of the eclipse (cyan), the CHAMP passes considered and the latitude of the magnetic equator (solid line). The bold parts of pass A4 indicate the location of the FAC regions. Contours indicate the expected shadow distribution at the time of the dip equator crossings of passes A3 and A4 (green and blue, respectively).

On pass A4 we encounter again an inter-hemispheric current from north to south. According to Yamashita and Iyemori (2002) this is a common feature of the morning sector during June solstice, but in this case the intensity is much stronger than reported in their study.

From Fig. 10 we can read that at the time of pass A4 the eclipse (blue contours) just pass the noon meridian. This means, the southern hemispheric illumination is strongly reduced. With that the seasonal imbalance is further enhanced. We regard this as the reason for the strong inter-hemispheric current.

\section{Summary}

We have presented the first satellite-based study of ionospheric current modifications which might be caused by midlatitude solar eclipses. This offers the opportunity to monitor also the inter-hemispheric field-aligned currents.

Two events occurring at very different conditions were considered, one eclipse at June solstice where CHAMP sampled the morning sector and another at the September equinox with CHAMP sampling the noon sector.

The major effects in both cases resemble seasonal features that are induces by the blocking of the sun. During the September event we observe FACs from the southern to the Northern Hemisphere comparable to that of the June solstice. Unexpected is the opposite current after the end of the eclipse which is obviously needed to bring the hemispheric current systems back into balance.

During the June eclipse a larger variety of eclipse related currents has been observed in the morning sector. Main feature is a meridional current system most probably driven by the F-region dynamo. Surprisingly, the observed current direction implies strong eastward equatorial winds, although westward zonal winds are expected at this time of the day. We suggest that this reverse wind may be caused by the delayed wind reversal from night to day time conditions due to the eclipse.

An intense north-south FAC is observed when the core shadow passes the noon meridian. Such an inter-hemispheric current in the morning sector is typical for June solstice. The larger intensity is probably due to the enhancement of the hemispheric difference by the solar eclipse in the south.

The presented results have helped to answer some of the open questions concerning the eclipse effects on the current systems. More such studies are, however, needed for revealing the significance of the results.

Acknowledgements. The operational support of the CHAMP mission by the German Aerospace Center (DLR) and the financial support for the data processing by the Federal Ministry of Education and Research (BMBF) are gratefully acknowledged. The work of A. T. Tomás is financed by a DFG (Deutsche Forschungsgemeinschaft) Research Fellowship.

Topical Editor M. Pinnock thanks A. Chulliat and R. Stening for their help in evaluating this paper. 


\section{References}

Chulliat, A., Blanter, E., Le-Mouël, J.-L., and Shnirman, M.: On the seasonal asymmetry of the diurnal and semidiurnal geomagnetic variations, J.Geophys. Res., 110, A05301, doi:10.1029/2004JA010551, 2004.

Drob, D. P., Emmert, J. T., Crowley, G., et al.: An Empirical Model of the Earth's Horizontal Wind Fields: HWM0,7, J. Geophys. Res., 113, A12304, doi:10.1029/2008JA013668, 2008.

Fukushima, N.: Field-aligned currents in the magnetosphere, Geof. Int., 30(4), 241-248, 1991.

Heelis, R. A.: Electrodynamics in the low and middle latitude ionosphere: a tutorial, J. Atmos. Solar-Terr. Phys., 66, 825-838, doi:10.1016/j.jastp.2004.01.034, 2004.

Korte, M., Lühr, H., Förster, M., and Haak, V.: Did the solar eclipse of August 11, 1999, show a geomagnetic effect, J. Geophys. Res., 106(A9), 18563, doi:10.1029/2001JA900006, 2001.

Lühr, H., Rother, M., Maus, S., Mai, W., and Cooke, D.: The diamagnetic effect of the equatorial Appleton anomaly: Its characteristics and impact on geomagnetic field modeling, Geophys. Res. Lett., 30(17), 1906, doi:10.1029/2003GL017407, 2003.

Lühr, H. and Maus, S.: Direct observation of the F region dynamo currents and the spatial structure of the EEJ by CHAMP, Geophys. Res. Lett., 33, L24102, doi:10.1029/2006GL028374, 2006.

Lühr, H., Rother, M., Häusler, K., Alken, P., and Maus, S.: The influence of nonmigrating tides on the longitudinal variation of the equatorial electrojet, J. Geophys. Res., 113, A08313, doi:10.1029/2008JA013064, 2008.
Maus, S. and Lühr, H.: A gravity-driven electric current in the Earth's ionosphere identified in CHAMP satellite magnetic measurements, Geophys. Res. Lett., 33, L02812, doi:10.1029/2005GL024436, 2006.

Maus, S., Rother, M., Stolle, C., Mai, W., Choi, S., Lühr, H., Cooke, D., and Roth, C.: Third generation of the Potsdam Magnetic Model of the Earth (POMME), Geochem. Geophys. Geosyst., 7, Q07008, doi:10.1029/2006GC001269, 2006.

Onwumechili, C. A.: The Equatorial Electrojet, Gordon and Breach Science Publishers, Amsterdam, Netherlands, 159-251, 1997.

Richmond, A. D.: Ionospheric Electrodynamics using magnetic apex coordinates, J. Geomag. Geoelec., 47, 191-212, 1995.

Risbeth, H.: The F-layer dynamo, Planet. Space Sci., 19, 263-267, 1971.

Tomás, A. T., Lühr, H., Rother, M., Manoj, C., Olsen, N., and Watari, S.: What are the influences of solar eclipses on the equatorial electrojet?, J. Atmos. Solar-Terr. Phys., 70, 1497-1511, doi:10.1016/j.jastp.2008.05.009, 2008.

Van Sabben, D.: Magnetospheric currents associated with the N-S asymmetry of the $S_{q}$, J. Atmos. Terr. Phys., 28, 965-981, 1966.

Yamashita, S. and Iyemori, T.: Seasonal and local time dependences of the inter-hemispheric field-aligned currents deduced from the Ørsted satellite and the ground geomagnetic observations, J. Geophys. Res., 107(A11), 1372, doi:10.1029/2002JA009414, 2002. 